\title{
Factors Influencing Tumor Response to Photodynamic Therapy Sensitized by Intratumor Administration of Methylene Blue
}

\author{
Timothy M. Baran, BS ${ }^{1}$, Benjamin R. Giesselman, BS ${ }^{2}$, Rui Hu, $\mathrm{PhD}^{3}$, Merrill A. Biel, MD, \\ $\mathrm{PhD}^{4}$, and Thomas H. Foster, $\mathrm{PhD}^{1,2}$ \\ ${ }^{1}$ Institute of Optics, University of Rochester, Rochester, NY USA 14627 \\ ${ }^{2}$ Department of Imaging Sciences, University of Rochester Medical Center, Rochester, NY USA \\ 14642 \\ 3Department of Biostatistics and Computational Biology, University of Rochester Medical Center, \\ Rochester, NY USA 14642
}

${ }^{4}$ Minneapolis ENT Research Foundation, Minneapolis, MN USA 55404

\begin{abstract}
Background and Objective-We examined tumor response to methylene blue (MB)-mediated photodynamic therapy (PDT) in a murine tumor model. The goal was to investigate the effects of drug-light interval (DLI), injection vehicle, and fluence on tumor destruction. Fluorescence and reflectance spectroscopy informed our understanding.
\end{abstract}

Materials and Methods-EMT6 tumor cells were implanted intradermally on the backs of female BALB/c mice and grown to 4-mm diameter. Mice were given a $35 \mu \mathrm{L}$, single site, intratumor injection of $500 \mu \mathrm{g} / \mathrm{mL}$ MB administered in either a water or a $5 \%$ ethanol-5\% Cremophor-90\% saline vehicle. PDT was begun either immediately or after a 1-hour DLI with a fluence rate of $60 \mathrm{~mW} / \mathrm{cm}^{2}$. Each animal received a fluence of 240 or $480 \mathrm{~J} / \mathrm{cm}^{2}$. Fluorescence and reflectance spectra were captured before and during irradiation.

\begin{abstract}
Results-A protocol consisting of the Cremophor-based vehicle, 0 DLI, and a fluence of $480 \mathrm{~J} /$ $\mathrm{cm}^{2}$ was the most effective, with a $55 \%$ cure rate as measured by no evidence of tumor 90 days after PDT. Use of the water vehicle with this fluence and DLI reduced the cure rate to $20 \%$. Reducing the fluence to $240 \mathrm{~J} / \mathrm{cm}^{2}$ similarly reduced treatment efficacy with 0 and 1 -h DLIs. Univariate Cox proportional hazards analysis identified increased fluence, 0 vs. 1-h DLI, and the Cremophor vs. water vehicle as highly significant independent predictors of long term tumor control ( $\mathrm{p}<0.01$ in each case). Multivariate analysis with model selection revealed fluence and injection vehicle as the best predictors of survival hazards. Fluorescence spectroscopy in vivo showed that MB fluorescence decreased monotonically during a 2-h dark interval but was restored by irradiation. Reflectance spectroscopy revealed that MB at this injected concentration attenuates the treatment beam significantly.
\end{abstract}

Conclusion-Sensitizer delivery vehicle, drug-light interval, and fluence contribute significantly to the tumor response to MB-mediated PDT.

\section{Keywords}

fluorescence spectroscopy; methylene blue; photodynamic therapy; reflectance spectroscopy

Correspondence to: Thomas H. Foster University of Rochester Department of Imaging Sciences 601 Elmwood Ave., Box 648 


\section{INTRODUCTION}

Photodynamic therapy (PDT) is an emerging treatment that has been used to treat malignant, premalignant, and benign conditions $[1,2]$. It has received health regulatory agency approval in many countries throughout the world, and clinical trials continue to establish new applications. Among these, an interesting and pressing application of PDT is in treating malignant tumors in patients that have not responded to more traditional therapies or which have recurred and may be otherwise untreatable [3,4].

A key component of PDT is the photosensitizer. We are investigating methylene blue (MB), an FDA-approved phenothiazine dye that is generally used to treat methemoglobinemia [5] and has been used successfully to stain intestinal metaplasia in Barrett's esophagus [6] and in the stomach to guide endoscopic biopsy [7]. It is inexpensive and is routinely available in hospital pharmacies. MB is also well known to be photodynamically active. Several studies have shown that MB mediated PDT (MB-PDT) can effectively inactivate viruses [8] and destroy bacteria [9]. Additionally, MB-PDT has been shown to be effective in destroying tumors in mice [10-12]. In humans, MB-PDT has been used to treat superficial tumors of the bladder [13], esophageal carcinomas [14], melanoma [15], and Kaposi's sarcoma [16] with no reported toxicities. Because it is biochemically modified to a colorless form [17], there is little if any systemic photosensitivity associated with MB.

Despite the potential advantages of MB and the opportunities presented by its FDA status, widespread availability, and low cost, there have not been systematic investigations of the effects of various treatment parameters on MB-PDT in animal models. Therefore, our intention in this study was to evaluate some of the factors that may influence the outcome of MB-PDT in a tumor model. The variables that we examined were drug-light interval, delivery vehicle, and fluence. Because MB is administered via direct intratumor injection, short drug-light intervals may be exploited without sacrificing selectivity. The shortest possible interval permits delivery of the greatest fluence in a treatment session of a given duration. The vehicle in which the photosensitizer is delivered may also have an impact on the outcome of the treatment insofar as it influences the homogeneity of intratumor distribution. Orth et al.[10] described inhomogeneous intratumor distribution of MB following injection using an aqueous vehicle. In previous studies with the photosensitizer Pc 4 , we demonstrated relatively uniform distributions with a Cremophor-ethanol-saline injection vehicle [18]. Finally, the fluence is another obvious factor influencing the treatment outcome. Anticipating an eventual clinical trial, we chose to limit the total treatment time from injection to the end of irradiation to approximately two hours. This constrained the maximum fluence for a given irradiance. The comparison of these factors was accomplished through a combination of spectroscopic studies in vitro and in vivo, imaging of MB fluorescence in freshly excised tumors, and long term tumor control trials in an intradermal murine tumor model.

\section{METHODS}

\section{Tumor Model}

Intradermal (ID) mouse mammary EMT6 tumors were initiated on the backs of female $\mathrm{BALB} / \mathrm{c}$ mice by ID injection of $10^{6}$ cells. Tumor growth was monitored daily. For a period of approximately 2 weeks prior to PDT and spectroscopy, mice were fed a chlorophyll-free diet prepared using the recipe of Holmes et al. [19]. Tumors were treated when volumes reached approximately $25-40 \mathrm{~mm}^{3}$. All experiments were conducted according to the institutional guidelines of the University of Rochester Medical Center and approved by the University Committee on Animal Resources. 


\section{Photosensitizer Administration and Light Treatment}

Sterile, clinical grade MB (10 mg/mL, American Regent, Shirley, NY) was obtained from the pharmacy at Strong Memorial Hospital, University of Rochester. This was diluted 1:20 in $\mathrm{ddH}_{2} \mathrm{O}$ to obtain a $500 \mu \mathrm{g} / \mathrm{mL}$ aqueous injection vehicle. A $5 \%$ ethanol-5\% Cremophor-90\% saline solution (Cremophor vehicle) containing the same $500 \mu \mathrm{g} / \mathrm{mL}$ concentration of $\mathrm{MB}$ was created by diluting $50 \mu \mathrm{L}$ of the sterile $\mathrm{MB}$ stock in $850 \mu \mathrm{L}$ of $0.9 \%$ saline and adding this to $100 \mu \mathrm{L}$ of a 1:1 solution of Cremophor and ethanol. Either the water or the Cremophor vehicle was administered in a $35 \mu \mathrm{L}$ dose via intratumor (IT) injection at a single site using a 29-gauge needle. Either immediately (0 drug-light interval) or after $1 \mathrm{~h}, 667 \mathrm{~nm}$ PDT treatment light from a diode laser (Power Technology, Alexander, AR) was delivered at $60 \mathrm{~mW} / \mathrm{cm}^{2}$ using the off-surface delivery probe shown in Figure 1. Fluences of 240 or $480 \mathrm{~J} / \mathrm{cm}^{2}$ were delivered, and the total treatment time (from MB injection to the end of irradiation) was constrained to approximately $2 \mathrm{~h}$. Irradiation was interrupted every $60 \mathrm{~J} / \mathrm{cm}^{2}(16 \mathrm{~min} 40 \mathrm{~s}$ ) for approximately $6 \mathrm{~s}$ in order to perform spectroscopy as described immediately below. Control animals received the same $35 \mu \mathrm{L}$ IT injection of MB with either the water or Cremophor vehicle but were not irradiated.

\section{Light Delivery and Spectroscopic Measurements}

Treatment light, excitation light for fluorescence spectroscopy, and broadband white light for reflectance spectroscopy were delivered to the surface of the tumor via a custom, offsurface probe shown in Figure 1. The probe consisted of a central GRIN-terminated fiber for delivery of treatment light and two SMA-terminated fibers, one of which served as a source for delivery of fluorescence excitation and broadband light while the other collected fluorescence and reflectance from the tumor. During a treatment session, PDT irradiation was interrupted every $60 \mathrm{~J} / \mathrm{cm}^{2}$ with a computer controlled in-line shutter (Mikropack, Ostfildern, Germany), and fluorescence excitation and broadband light were routed sequentially via an optical switch (Piezosystem Jena, Hopedale, MA) to the source fiber of the probe. Fluorescence excitation was performed with $639 \mathrm{~nm}$ light from a diode laser $(\mathrm{Oz}$ Optics, Ottawa, Ontario, Canada). The broadband reflectance source was a tungsten-halogen lamp (Avantes, Broomfield, CO). Fluorescence and reflectance spectra were acquired by dedicated, TE-cooled, 16 bit spectrometers (B \& W Tek, Newark, DE) using integration times of 5 and $0.5 \mathrm{~s}$, respectively. The fluorescence detection path included a dichroic mirror (FF669-Di01, Semrock, Rochester, NY) and a long pass filter (LP02-664RU, Semrock) as shown in Figure 1. The source and detector fibers of the probe interrogated an overlapping 3 $\mathrm{mm}$-diameter area at the center of the treatment field. A custom LabVIEW program (National Instruments, Austin, TX) controlled the sequence of PDT irradiation and spectral acquisitions.

\section{Spectral Processing and Analysis}

Spectra were corrected by subtracting the background and then dividing by the measured, wavelength-dependent system response. Backgrounds were taken before each mouse was treated by integrating dark signals for the durations described above. System responses were acquired by reflecting the light from a NIST-traceable lamp (Model \# LS-1-CAL, Ocean Optics, Dunedin, FL) off of a diffuse reflectance standard (Part \# WS-1, Ocean Optics) into the probe collection fiber. The measured spectrum was then background subtracted and divided by the known lamp spectrum to obtain the system response. Fluorescence spectra were further corrected for the effects of tissue optical properties by dividing the fluorescence by reflectance spectra measured in the same wavelength range and in the same geometry [20].

After fluorescence spectra were acquired and corrected, they were fit using a singular value decomposition (SVD) algorithm based on the work of Press et al. [21]. The two basis 
spectra shown in Figure 2(a) were used in the fits. The first was the monomeric MB fluorescence spectrum, determined by measuring the fluorescence emission of $3.1 \mu \mathrm{M} \mathrm{MB}$ in a commercial fluorometer (Varian Eclipse, Palo Alto, CA). The sample was prepared in a 1:10 dilution of 10\% Liposyn (Abbott Laboratories, North Chicago, IL) in de-ionized water in order to more closely replicate conditions in vivo. The other basis was created in order to fit the long wavelength fluorescence. This was done by slowly increasing the concentration of MB from 3 to approximately $200 \mu \mathrm{M}$ and acquiring fluorescence spectra as shown in Figure 2(b). The spectra were then normalized to their maximum value and shifted so their maxima aligned. The lowest concentration spectrum was then subtracted from the highest concentration spectrum in order to isolate the long wavelength fluorescence. This difference was then fitted with a Gaussian in order to generate the dimer basis spectrum. In addition to the basis spectra, a series of 61 Fourier terms was also used in fitting to account for unknown possible contributions to the measured fluorescence in vivo. These Fourier terms were given a smaller weight in the fitting in order to favor the basis spectra.

Fitting followed the SVD scheme found in MATLAB (Mathworks, Natick, MA). However, we allowed the basis functions to shift a few nanometers in either direction in order to account for apparent absorption-induced changes in fluorescence that were not corrected by the division by reflectance.

\section{Imaging MB Fluorescence in Freshly Excised EMT6 Tumors}

Freshly excised tumors from several mice were sectioned in half and imaged using a stereofluorescence microscope (Model SMZ1500, Nikon Instruments Inc., Melville, NY) to assess intratumor distribution of MB. Mice were given an IT injection of $35 \mu \mathrm{L}$ of $500 \mu \mathrm{g} / \mathrm{mL}$ MB in either the water or Cremophor vehicle. They were then immediately sacrificed and the tumors excised. Tumors were then sliced in half and placed on the stage of microscope. Imaging was performed using $639 \mathrm{~nm}$ laser excitation (Oz Optics, Ottawa, Ontario, Canada), routed to the microscope with a liquid light guide, and a custom filter cube optimized for MB (Micro Video Inst., Avon, MA). Images were acquired at 12 bits using a Photometrics CoolSNAPHQ CCD (Roper Scientific, Inc., Trenton, NJ). An image of a homogeneous methylene blue solution served as a system response function, and each subsequent image was divided by this image to eliminate non-uniformities due to illumination and collection optics. Image analysis was performed using the Surface Plot tool in ImageJ (NIH; URL: http://rsb.info.nih.gov/ij/).

\section{Tumor Response Assay and Statistics}

Tumor dimensions along three axes were measured daily following PDT. The tumor volume was computed assuming an ellipsoidal shape. Mice were removed from the study if the tumor grew to twice the volume measured at the time of irradiation. Cures were defined as no palpable tumor 90 days after PDT. Uni- and multivariate statistical analysis of tumor responses was performed using a Cox proportional hazards model implemented in the $\mathrm{R}$ package survival (http://cran.r-project.org/web/packages/survival/index.html). Model selection in the multivariate analysis was done using an Akaike information criterion (AIC).

\section{RESULTS}

Fluorescence and reflectance spectra were acquired from MB-sensitized EMT6 tumors in vivo immediately before, at defined intervals during, and immediately after irradiation. Measured fluorescence spectra were fit with an SVD algorithm and two basis spectra, as described in Methods. The results of this fitting can be seen in Figure 3. Figure 3(a) shows a representative fit to a spectrum acquired immediately after IT injection of $500 \mu \mathrm{g} / \mathrm{mL}$ MB in the water vehicle. The spectrum is fit well with a superposition of the monomer and dimer 
bases. In this case the dimer/monomer ratio was 0.26 , but there was considerable tumor-totumor variation in this pre-irradiation ratio. The fluorescence spectrum of the same tumor after irradiation with $480 \mathrm{~J} / \mathrm{cm}^{2}$ is shown in Figure 3(b). The overall fluorescence magnitude is increased relative to the pre-irradiation spectrum, and there is an increased contribution of the dimer basis to the fit. In this tumor, the post-irradiation dimer/monomer ratio increased to 0.52 , but this also was not a general trend.

Figure 4(a) shows a series of fluorescence spectra acquired in vivo from a tumor that received a $35 \mu \mathrm{L}$ IT injection of $500 \mu \mathrm{g} / \mathrm{mL}$ MB in the Cremophor vehicle. Fluorescence was measured every 15 minutes after injection for a period of two hours in the absence of irradiation. As can be seen, the measured fluorescence decreases monotonically after injection during this interval. Figure 4(b) summarizes this trend in mice monitored for a period of one hour, after which seven mice were irradiated with $667 \mathrm{~nm}$ light at $60 \mathrm{~mW} / \mathrm{cm}^{2}$. In the irradiated mice, the amplitude of MB fluorescence was progressively restored and in some cases exceeded the pre-irradiation levels, while fluorescence from the unirradiated tumors continued to decrease. This irradiation-induced increase suggests that the measured loss of fluorescence during the dark interval is not the result of clearance of MB from the tumor. Rather, it likely originates in the biochemical modification to a colorless form as described by DiSanto and Wagner [17].

To assess the intratumor distribution of MB following IT injection at a single site, mice were sacrificed immediately after injection, tumors were excised, sectioned in half, and placed on the stage of the stereofluorescence microscope. Representative images and image analysis are shown in Figure 5 for the Cremophor $(a, c)$ and water $(b, d)$ injection vehicles. Tumors injected using the Cremophor vs. water vehicle show a greater degree of MB fluorescence homogeneity. Image analysis revealed that fluctuations in pixel intensities were more pronounced (6- vs. $\sim 2.5$-fold) in tumors injected with the water vehicle. Nearly $10 \%$ of the pixels in the "aqueous" image were below a threshold set at twice the background counts measured from control tumors under identical image acquisition conditions. In the "Cremophor" images, fewer than $0.5 \%$ of pixels were below this threshold.

Results of the tumor control studies are illustrated in the Kaplan-Meier plots of Figure 6. As described in Methods, tumor volume doubling was the end point used as a measure of treatment failure. Cures were defined as no evidence of tumor 90 days after PDT. All mice received the same $35 \mu \mathrm{L}$ injection of $500 \mu \mathrm{g} / \mathrm{mL}$ MB administered in either the water or Cremophor vehicle. In addition to injection vehicle, we investigated responses to 480 vs. $240 \mathrm{~J} / \mathrm{cm}^{2}$ fluence. Based on anticipated clinical constraints, we limited the duration of the procedure (from time of injection to end of irradiation) to approximately 2 hours. Thus, we evaluated protocols in which irradiation was initiated immediately after sensitizer injection in order to deliver the maximum fluence within this 2 hour interval. Among these protocols, the most successful combined the Cremophor vehicle, a zero drug-light interval, and a fluence of $480 \mathrm{~J} / \mathrm{cm}^{2}$. A long-term cure rate of 55\% (5 of 9) was achieved under these conditions. Univariate statistical analysis of all of the protocols using a Cox proportional hazards model revealed that increased fluence $(\mathrm{p}=0.00042)$, decrease in the drug-light interval $(\mathrm{p}=0.0013)$, and the use of a Cremophor vs. water vehicle $(\mathrm{p}=0.009)$ were all statistically significant predictors of long-term tumor response, with $\mathrm{p}$-values $<0.05$ for each of these treatment parameter comparisons. From a multivariate Cox proportional hazards analysis and model selection based on AIC, a combination of increased fluence $(\mathrm{p}=0.001)$ and use of the Cremophor vehicle $(\mathrm{p}=0.027)$ emerged as the best predictor of treatment outcome $(\mathrm{p}<0.05)$.

As mentioned above, reflectance spectra were also acquired before, during, and immediately after PDT. In addition to allowing for correction of the fluorescence spectra, reflectance 
provides a measure of tissue optical properties. Figure 7 shows a series of three reflectance spectra taken in vivo from the same tumor prior to injection of MB, immediately after injection but before the start of irradiation, and immediately post-irradiation with $480 \mathrm{~J} / \mathrm{cm}^{2}$. Particularly noteworthy is the greatly reduced reflectance (i.e. higher absorption) at the 667 $\mathrm{nm}$ treatment wavelength from the presence of MB. After irradiation only a modest increase in reflectance is observed. Thus MB at this injection concentration significantly increases tissue absorption at the PDT treatment wavelength, and this will be a factor in treatment planning for larger tumors. We are currently quantifying this effect and evaluating its consequences.

\section{DISCUSSION}

Our experiments investigated effects of intratumor sensitizer delivery vehicle, the drug-light interval, and the fluence as factors capable of influencing the response of tumors to MBPDT in vivo. Results illustrate that indeed attention to each of these treatment variables contributes to long term tumor control in this model. Specifically, the Cremophor vs. water vehicle, a 0 vs. $1 \mathrm{~h}$ drug-light interval, and increased fluence all independently predicted for reduced survival hazard.

The increased efficacy provided by the Cremophor vehicle is likely a result of a more homogeneous intratumor distribution of MB following a single injection, which is illustrated in Figure 5. Fluorescence imaging of freshly excised EMT6 tumors showed reduced fluctuations in pixel intensities and fewer pixels with very low levels of MB fluorescence in tumors injected with the Cremophor vs. water vehicle. Orth et al. also reported heterogeneous intratumor distribution of MB following multiple injections of an aqueous vehicle in larger tumor volumes [10]. Methylene blue is water soluble, thus the improved intratumor distribution with the EtOH-Cremophor injection may be a result of the solvent drag phenomenon, discussed recently by Boiy et al. in the context of topical formulations for hypericin [22]. We note also that the injection of $500 \mu \mathrm{g} / \mathrm{mL}$ MB in the Cremophor vehicle resulted in statistically significant EMT6 tumor growth delay relative to untreated controls (Fig. 6, p < 0.001). The role of this modest dark effect in long term tumor control is uncertain. Further, we have emphasized the role of the vehicle with respect to macroscopic intratumor distribution, but we have not considered the effects of vehicle on intracellular distribution of $\mathrm{MB}$, a problem which is difficult to address in whole tumors following sensitizer administration in vivo.

Effects of the drug-light interval in intratumor MB-PDT have not been reported previously. The reduction of MB to a colorless form in vivo probably precludes systemic administration, but our fluorescence spectroscopy results, presented in Figure 4, show for the first time that a progressive loss of MB fluorescence occurs even after direct intratumor injection. This may provide a rationale for a very short drug light interval. As shown also in Figure 4, however, the MB fluorescence is restored in response to irradiation. Our statistical analysis suggests that even with this restoration, there is an advantage to a 0 vs. 1-h drug-light interval. In the case of intratumor sensitizer administration, long drug-light intervals are not needed in order to achieve selectivity. It is still desirable of course to allow enough time for the sensitizer to distribute throughout the tumor volume. The images of Figure 5 show that this happens immediately after injection of a $35 \mu \mathrm{L}$ volume, indicating that fluid pressure as opposed to passive diffusion drives the rapid transport of MB. Finally, in the anticipated clinical setting, it is desirable to limit the total treatment time. The 0 drug-light interval enables delivery of the maximum fluence in a given treatment duration. Taking all of this into account, if treatment time is constrained, a protocol including an efficient vehicle and the shortest possible drug-light interval will provide the highest fluence and the greatest efficacy. Other factors, specifically treatment irradiance and the use of more than one PDT 
treatment, which were evaluated by Orth et al. [10], have not been considered here but may certainly contribute, as well.

The in vivo fluorescence and reflectance spectra shown in Figures 3, 4, and 7 are, to the best of our knowledge, the first such data to be reported in the literature. As illustrated in Figure 3 , MB fluorescence from tumors could be fit consistently using a superposition of the two basis spectra shown in Figure 2. These bases were constructed from cuvette measurements over a wide range of $\mathrm{MB}$ concentrations and are attributed to monomer and dimer/ aggregated forms. Tumor spectra generally contained contributions from both species, and it was not unusual for their relative contributions to change in response to PDT, as was the case for the tumor represented in Figure 3. There was, however, considerable heterogeneity in the pre-irradiation dimer/monomer ratio extracted from fits to spectra. Further, no significant trends were discernable in the dimer/monomer ratio changes induced by irradiation. In the case shown in Figure 3, the amplitudes of both the monomer and the dimer contributions to the spectrum increased in response to irradiation, thereby ruling out a simple interpretation based on a shifting equilibrium between monomers and aggregates. MB is capable of complex chemistry, which is reflected in the following equations [23],

$$
\begin{gathered}
2 \mathrm{MB} \stackrel{\mathrm{K}_{\mathrm{D}}}{\rightleftarrows}(\mathrm{MB})_{2} \\
(\mathrm{MB})_{2} \stackrel{\mathrm{k}_{1}}{\longrightarrow} \mathrm{MB} \cdot+\mathrm{MB}^{2+} \\
\mathrm{MB} \cdot+\mathrm{O}_{2} \stackrel{\mathrm{k}_{2}}{\longrightarrow} \mathrm{O}_{2}{ }^{-}+\mathrm{MB}
\end{gathered}
$$

where Eq. (1) represents the equilibrium between MB monomers (MB) and dimers, $(\mathrm{MB})_{2}$, with equilibrium constant $\mathrm{K}_{\mathrm{D}}$. Equation (2) represents the creation of $\mathrm{MB}$ radicals from ground state dimers at a rate $\mathrm{k}_{1}$ proportional to the laser excitation, with MB. and MB. ${ }^{2+}$ indicating the semi-reduced and semi-oxidized radical forms, respectively. Finally, Equation (3) represents the formation of superoxide, $\mathrm{O}_{2}{ }^{-}$, and ground state $\mathrm{MB}$ monomer at the rate $\mathrm{k}_{2}$ from the semi-reduced MB radical in the presence of molecular oxygen. Thus, the creation and dissociation of aggregates in vivo is likely to be a complex process, especially in the presence of heterogeneous and possibly dynamic tumor oxygen concentrations. The interpretation of MB fluorescence before, during, and after PDT is therefore a rich area for further study. As indicated in Eqs. (2) and (3), it is entirely likely that Type I processes contribute to tumor destruction with MB-PDT. Finally, the reflectance spectra shown in Figure 7 reveal an important aspect of PDT performed with relatively high intratumor concentrations. MB at the concentration injected in our study significantly attenuates the transmission of $667 \mathrm{~nm}$ treatment light, indicated by the vertical arrows in the Figure. This attenuation is not appreciably reduced by irradiation-induced photodegradation of MB. This effect on tumor optical properties will have to be taken into account in treatment planning.

A single intratumor MB administration in the Cremophor vehicle followed immediately by irradiation at the relatively high fluence of $480 \mathrm{~J} / \mathrm{cm}^{2}$ yielded $55 \%$ long term cures in this model, as shown in the Kaplan-Meier curves of Figure 6. It is likely that further attention to treatment optimization will improve these outcomes. In the near term while other promising photosensitizers are subjected to rigorous clinical evaluation leading to FDA approval, it is reasonable to consider MB-PDT as a treatment alternative in some patient populations. As 
noted in the Introduction, MB is approved by the US FDA and therefore could be used off label for PDT. It is safe, readily available, and, relative to other FDA approved PDT agents, extremely inexpensive. At our hospital pharmacy, a $10 \mathrm{~mL}$ vial of a sterile $10 \mathrm{mg} / \mathrm{mL}$ solution costs less than $\$ 3$. These factors combine to justify further investigation of this form of PDT.

\section{Acknowledgments}

This work was supported by NIH grants CA68409 and CA122093 awarded by the National Cancer Institute. The authors wish to thank Dr. Soumya Mitra for helpful discussions and for a careful reading of the manuscript.

Footnotes to the Title Page: This work was supported by NIH grants CA68409 and CA122093. MAB owns stock in Ondine Biopharma, which is commercializing a form of methylene blue PDT for treatment of periodontal disease.

\section{REFERENCES}

1. Brown SB, Brown EA, Walker I. The present and future role of photodynamic therapy in cancer treatment. Lancet Oncol. 2004; 5(8):497-508. [PubMed: 15288239]

2. O'Connor AE, Gallagher WM, Byrne AT. Porphyrin and nonporphyrin photosensitizers in oncology: preclinical and clinical advances in photodynamic therapy. Photochem Photobiol. 2009; 85(5):1053-1074. [PubMed: 19682322]

3. D'Cruz AK, Robinson MH, Biel MA. mTHPC-mediated photodynamic therapy in patients with advanced, incurable head and neck cancer: A multicenter study of 128 patients. Head Neck. 2004; 26(3):232-240. [PubMed: 14999798]

4. Lou PJ, Jager HR, Jones L, Theodossy T, Brown SG, Hopper C. Interstitial photodynamic therapy as salvage treatment for recurrent head and neck cancer. Br J Cancer. 2004; 91(3):441-446. [PubMed: 15238981]

5. Clifton J II, Leikin JB. Methylene Blue. Am J Ther. 2003; 10(4):289-291. [PubMed: 12845393]

6. Canto MIF, Setrakian S, Petras RE, Blades E, Chak A, Sivak MV. Methylene blue selectively stains intestinal metaplasia in Barrett's esophagus. Gastrointest Endosc. 1996; 44(1):1-7. [PubMed: 8836709]

7. Fennerty MB, Sampliner RE, McGee DL, Hixson LJ, Garewal HS. Intestinal metaplasia of the stomach: identification by a selective mucosal staining technique. Gastrointest Endosc. 1992; 38(6): 696-698. [PubMed: 1282115]

8. Jockusch S, Lee D, Turro NJ, Leonard EF. Photo-induced inactivation of viruses: Adsorption of methylene blue, thionine, and thiopyronine on Q $\beta$ bacteriophage. Proc Natl Acad Sci USA. 1996; 93(15):7446-7451. [PubMed: 8755492]

9. Usacheva MN, Teichert MC, Biel MA. The role of the methylene blue and toluidine blue monomers and dimers in the photoinactivation of bacteria. J Photochem Photobiol B. 2003; 71(2):87-98. [PubMed: 14705643]

10. Orth K, Beck G, Genze F, Ruck A. Methylene blue mediated photodynamic therapy in experimental colorectal tumors in mice. J Photochem Photobiol B. 2000; 57(3):186-192. [PubMed: 11154085]

11. Konig K, Bockhorn V, Dietel W, Schubert H. Photochemotherapy of animal tumors with the photosensitizer methylene blue using a krypton laser. J Cancer Res Clin Oncol. 1987; 113(3):301303. [PubMed: 3584221]

12. Orth K, Russ D, Beck G, Ruck A, Beger HG. Photochemotherapy of experimental colonic tumors with intra-tumorally applied methylene blue. Langenbck Arch Surg. 1998; 383(3):276-281.

13. Williams JL, Stamp J, Devonshire R, Fowler GJ. Methylene blue and photodynamic therapy of superficial bladder cancer. J Photochem Photobiol B. 1989; 4(2):229-232. [PubMed: 2512384]

14. Orth K, Ruck A, Stanescu A, Beger H. Intraluminal treatment of inoperable oesophageal tumors by intralesional photodynamic therapy with methylene blue. Lancet. 1995; 345(8948):519-520.

[PubMed: 7532255] 
15. Tardivo J, Giglio A, Paschaol L, Ito A, Baptista M. Treatment of melanoma lesions using methylene blue and RL50 light source. Photodiagnosis Photodyn Ther. 2004; 1(4):345-346.

16. Tardivo J, Giglio A, Paschaol L, Ito A, Baptista M. New photodynamic protocol to treat AIDSrelated Kaposi's sarcoma. Photomed Laser Surg. 2006; 24(4):528-531. [PubMed: 16942436]

17. DiSanto AR, Wagner JG. Pharmokinetics of highly ionized drug III: Methylene blue - blood levels in the dog and tissue levels in the rat following intravenous administration. J Pharm Sci. 1972; 61(7):1090-1094. [PubMed: 5044808]

18. Foster TH, Giesselman BR, Hu R, Kenney ME, Mitra S. Intratumor administration of the photosensitizer Pc 4 affords photodynamic therapy efficacy and selectivity at short drug-light intervals. Transl Oncol. 2010; 3(2):135-141. [PubMed: 20360938]

19. Holmes HJ, Kennedy JC, Pottier R, Rossi R, Weagle G. A recipe for the preparation of a rodent food that eliminates chlorophyll-based tissue fluorescence. J Photochem Photobiol B. 1995; 29(2): 199. [PubMed: 7472814]

20. Wu J, Feld MS, Rava RP. Analytical model for extracting intrinsic fluorescence in turbid media. Appl Opt. 1993; 32(19):3585-3595. [PubMed: 20829983]

21. Press, WH.; Teukolsky, SA.; Vetterling, WT.; Flannery, BP. Numerical Recipes in C: The Art of Scientific Computing. 2nd ed. Cambridge University Press; New York: 1992.

22. Boiy A, Roelandts R, de Witte PAM. Influence of application and formulation factors on the penetration of hypericin in normal mouse skin and UV induced skin tumors. J Photochem Photobiol B. 2007; 89(3):156-162. [PubMed: 17983765]

23. Junqueira HC, Severino D, Dias LG, Gugliotti MS, Baptista MS. Modulation of methylene blue photochemical properties based on adsorption at aqueous micelle interfaces. Phys Chem Chem Phys. 2002; 4(11):2320-2328. 


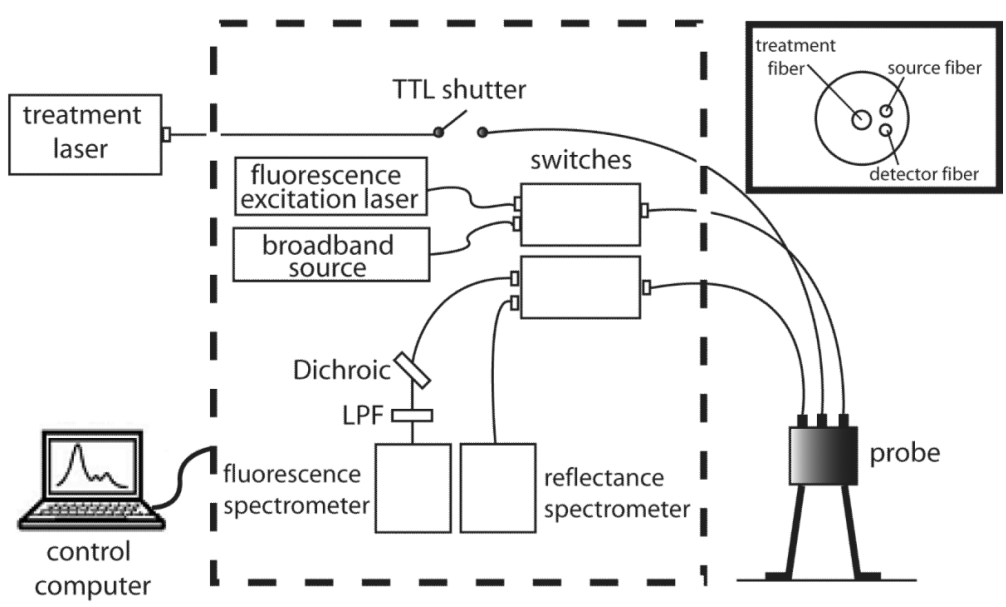

Figure 1.

Schematic of PDT treatment and spectroscopy system. Switches, sources, and spectrometers are computer controlled. A $664 \mathrm{~nm}$ long pass filter and a $669 \mathrm{~nm}$ dichroic mirror are used to filter out the excitation source in the fluorescence detection arm. Inset shows schematic of probe for delivery of treatment light, fluorescence excitation, and broadband white light and collection of spectra. The treatment fiber is a $400 \mu \mathrm{m}$ core diameter fiber terminated in a microlens diffuser $(\mathrm{NA}=0.39)$. The spectroscopy source and detector fibers have core diameters of 600 and $400 \mu \mathrm{m}$, respectively. Both have a numerical aperture of 0.22 . The probe is not drawn to scale. 

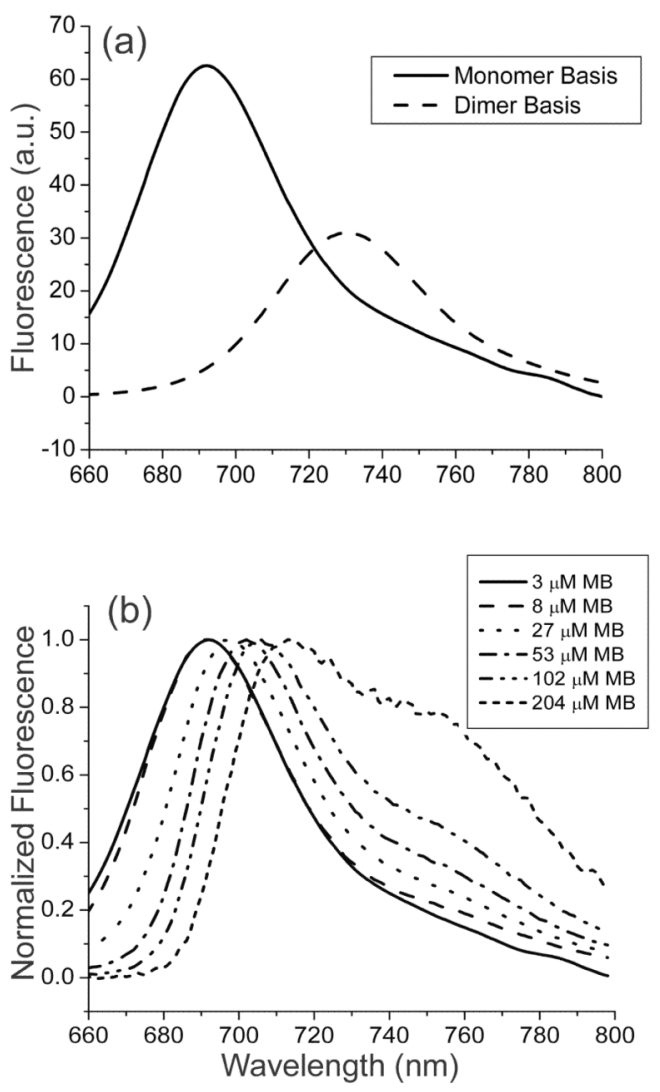

Figure 2.

(a) Bases used for SVD fitting, as determined by cuvette measurements made in a commercial fluorometer. Amplitude scaling is arbitrary and meant to illustrate relative contributions of bases to overall SVD fitting. (b) Normalized fluorescence spectra of MB measured in a commercial fluorometer illustrate shifted peak wavelength and growing contribution of long wavelength components with increasing MB concentration. 

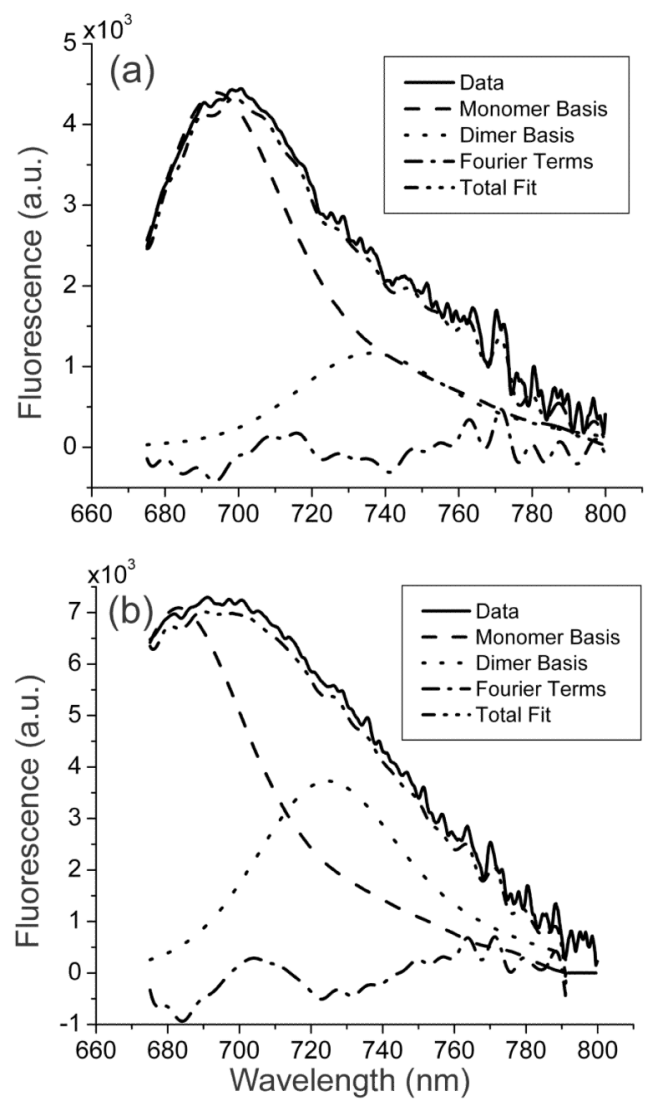

Figure 3.

Results of SVD fitting of a representative fluorescence spectrum taken from the same EMT6 tumor in vivo (a) immediately after IT injection of MB but before delivery of treatment light and (b) after conclusion of PDT $\left(480 \mathrm{~J} / \mathrm{cm}^{2}\right)$ under identical acquisition conditions, illustrating the increased magnitude of fluorescence and an increased presence of dimer fluorescence induced by irradiation. 


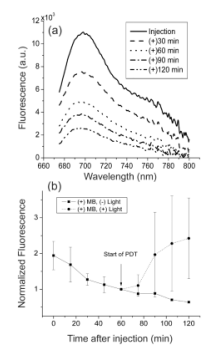

Figure 4.

(a) MB fluorescence spectra in vivo at various times following IT administration of MB in a 5\% EtOH, 5\% Cremophor, 90\% saline vehicle. Excitation wavelength was $639 \mathrm{~nm}$. (b) Magnitude of MB fluorescence before and during PDT, normalized to the onset of irradiation. All mice received $35 \mu \mathrm{L}$ of $500 \mu \mathrm{g} / \mathrm{mL}$ of $\mathrm{MB}$ in Cremophor based vehicle. Irradiated mice $(\mathrm{n}=7)$ received $240 \mathrm{~J} / \mathrm{cm}^{2}, 60 \mathrm{~mW} / \mathrm{cm}^{2}$ at $667 \mathrm{~nm}$. MB fluorescence in mice not irradiated $(n=2)$ continued to decrease from 60 to 120 min after injection. 

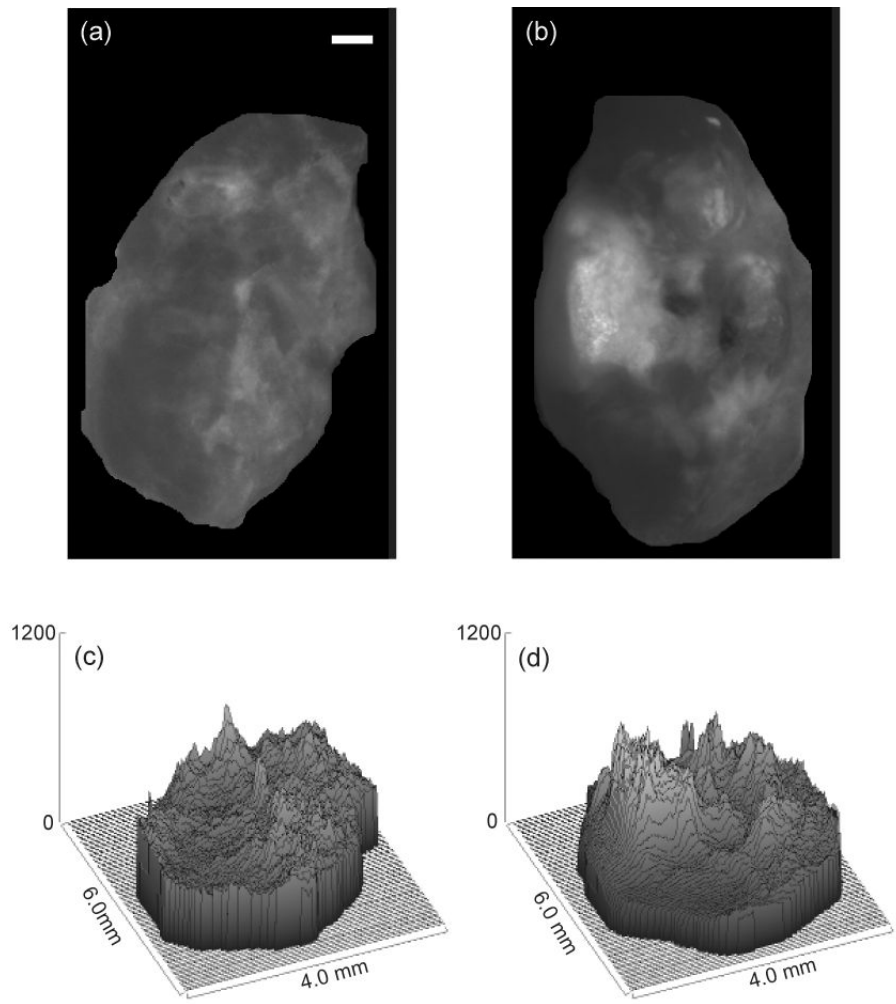

Figure 5.

MB fluorescence images of freshly sectioned EMT6 tumors acquired immediately after IT administration of $500 \mu \mathrm{g} / \mathrm{mL} \mathrm{MB}$ in the Cremophor (a) and water (b) injection vehicles. (c) and (d) are surface plots of pixel intensities from (a) and (b), respectively. The scale bar is $0.5 \mathrm{~mm}$. 


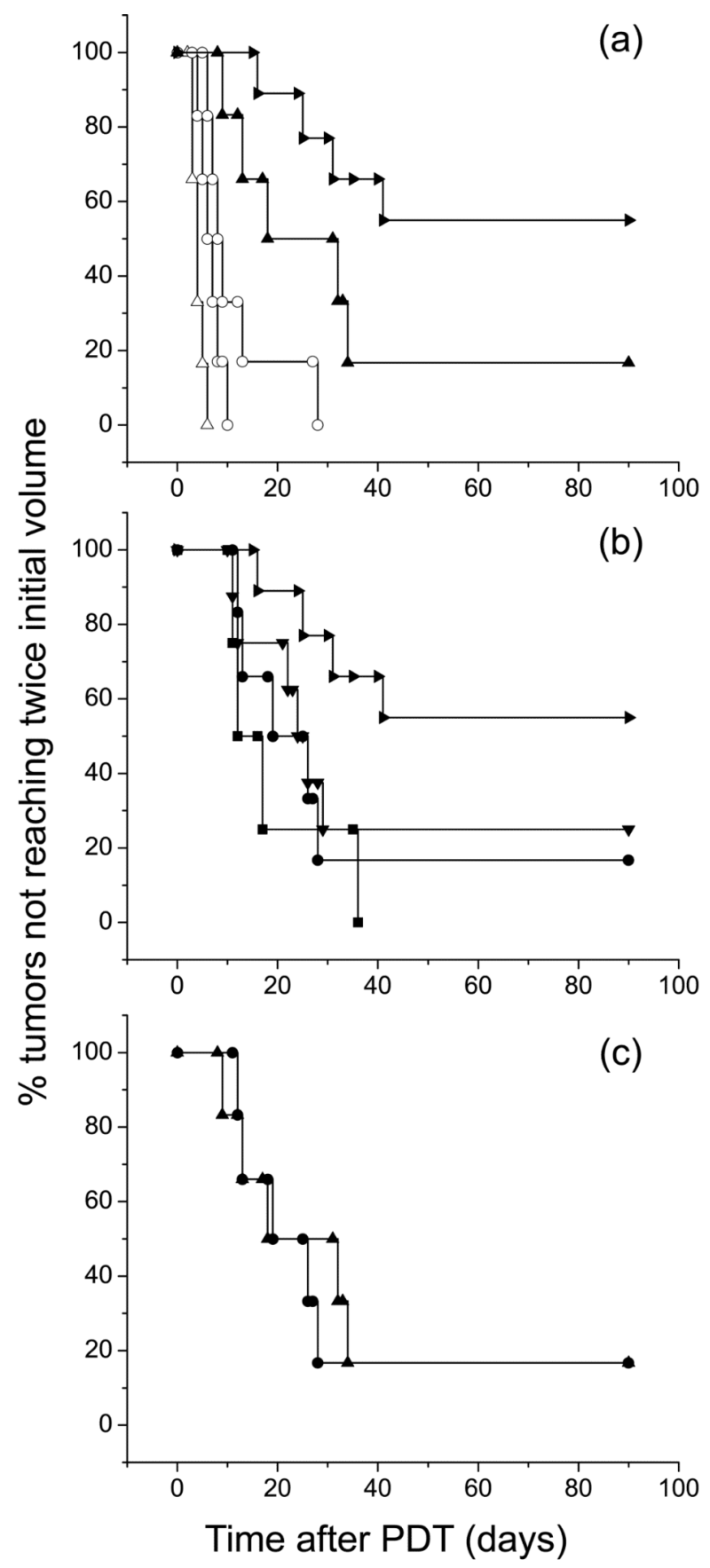

Figure 6.

Kaplan-Meier curves illustrating effects of (a) fluence, (b) sensitizer delivery vehicle, and (c) drug-light interval on response of EMT6 tumors to methylene-blue PDT in vivo. (a) $(\triangle)$ Drug and irradiation free control $(n=6) ;(\diamond)$ Drug only control, water vehicle $(n=6) ;(0)$ Drug only control, Cremophor vehicle $(n=6) ;(\boldsymbol{\Delta})$ Cremophor vehicle, 0 drug-light interval, $240 \mathrm{~J} / \mathrm{cm}^{2}(\mathrm{n}=6)$; ( ) Cremophor vehicle, 0 drug-light interval, $480 \mathrm{~J} / \mathrm{cm}^{2}$ (n=9); (b) (a) Water vehicle, $1 \mathrm{~h}$ drug-light interval, $240 \mathrm{~J} / \mathrm{cm}^{2}(\mathrm{n}=5) ;(\bullet)$ Cremophor vehicle, $1 \mathrm{~h}$ druglight interval, $240 \mathrm{~J} / \mathrm{cm}^{2}(\mathrm{n}=6)$; ( $\left.\boldsymbol{\nabla}\right)$ Water vehicle, 0 drug-light interval, $480 \mathrm{~J} / \mathrm{cm}^{2}(\mathrm{n}=10)$; (๖) Cremophor vehicle, 0 drug-light interval, $480 \mathrm{~J} / \mathrm{cm}^{2}(\mathrm{n}=9)$; (c) (•) Cremophor vehicle, 1 
h drug-light interval, $240 \mathrm{~J} / \mathrm{cm}^{2}(\mathrm{n}=6) ;$ ( $\mathbf{\Delta}$ ) Cremophor vehicle, 0 drug-light interval, $240 \mathrm{~J} /$ $\mathrm{cm}^{2}(\mathrm{n}=6)$. 


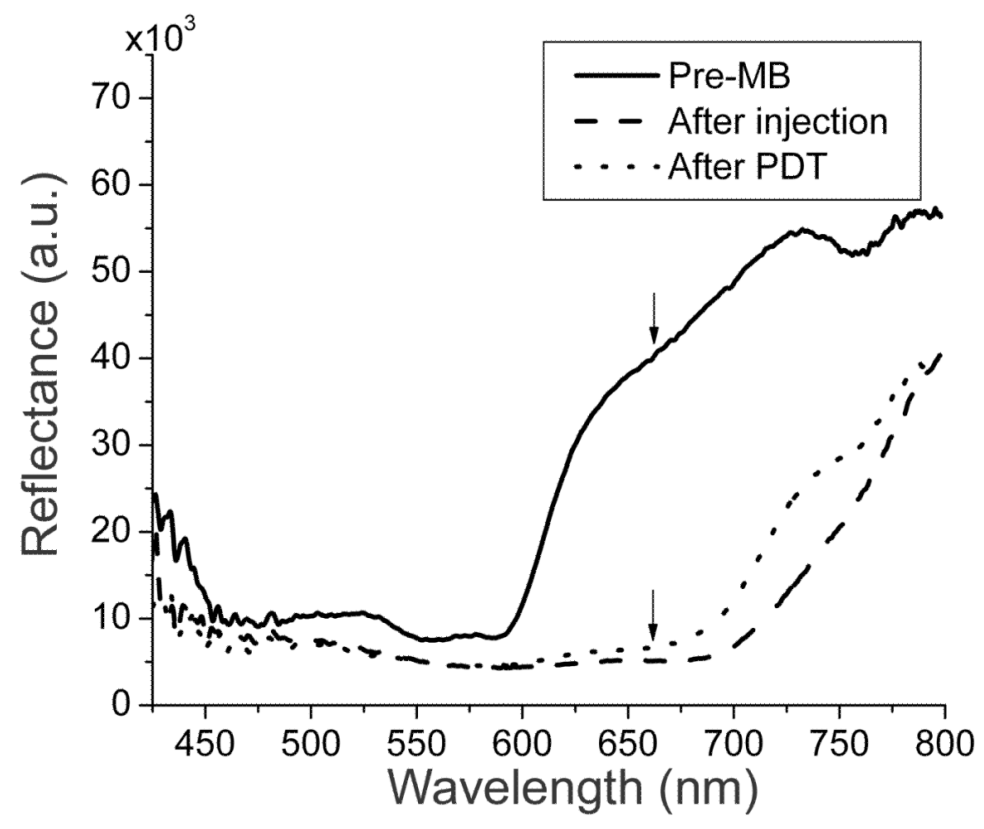

Figure 7.

Reflectance spectra measured from an EMT6 tumor before IT injection of MB, immediately after injection $(500 \mu \mathrm{g} / \mathrm{mL}, 35 \mu \mathrm{L})$, and immediately after irradiation with $480 \mathrm{~J} / \mathrm{cm}^{2}$. Post injection spectrum shows decreased reflectance (i.e. increased absorption) at the treatment wavelength (arrow) and negligible irradiation-induced increase in reflectance. 